\title{
Neural Network based Face Recognition System using Discrete Cosine Transform and Principal Component Analysis
}

\author{
C. Rajivegandhi \\ Department of Computer \\ Science \\ SRM University \\ Chennai, India
}

\author{
C. Sabarinathan \\ Department of Computer \\ Science \\ SRM University \\ Chennai, India
}

\author{
B. Abinaya \\ Department of Computer \\ Science \\ SRM University \\ Chennai, India
}

\begin{abstract}
This paper deals with the implementation of Neural Network based face recognition system. As we know that face recognition system is one of the biometric information processing which has speed up in the last few decades. The developed algorithm for the face recognition system originates an image based approach, which uses the TwoDimensional Discrete Cosine Transform (2D-DCT) to compress image, and then Self Organizing Map (SOM) Neural Network to recognize the face and its simulated in MATLAB. With the help of 2D-DCT the image vectors are extracted and these vectors sends to the neural network classifier which is developed using self organizing map, algorithm to recognize trained faces, faces with variations in expressions, changes of illumination, upto certain degrees. The alternate way of the same face recognition system is developed with the help of principle component analysis (PCA) instead of Two Dimensional Cosine Transform and Self-Organizing Map Neural Network to recognize the faces. In this proposed algorithm we use unsupervised single neural network as a classifier for both Two Dimensional Discrete Cosine Transform (2D-DCT) and Principal Component Analysis (PCA).
\end{abstract}

\section{Keywords}

Artificial Neural Network, Self Organizing Map, Principal Component Analysis, Two Dimensional Discrete Cosine Transform, Unsupervised.

\section{INTRODUCTION}

Recognition [3,5] of faces has a vital role as it is used in commercial and law enforcements, such us forensic identification, access control, human computer interactions, and border close observation. In the recent past and now there is a growing interest in image processing algorithms that try to analyze the image content (face). These algorithms are used by applications which require automatic detection of human faces in images with a complex background.

However, a face can have many variations in a scene. It can be anywhere in the image, have a variety of sizes, poses and illuminations. Many techniques are available which apply face recognition that work efficiently in constrained environment. The most common approach for face recognition is artificial neural networks. In this paper the algorithm applied to the problem of face detection belong to the concept of biometrics. Biometrics refers to the unmanned acknowledgement of individuals based on their physiological and/or behavioral characteristics.

A biometrics is a pattern acknowledgement system that operates by gaining biometric data from a novel by withdrawing a set of attributes from the data acquired, and this is compared with the feature set against the template set in the database In this paper, The proposed recognition system uses artificial neural network (ANN).WISARD is a single layer adaptive network which contains a separate network for each stored individuals is used for face recognition in Artificial Neural Network.

\section{LITERATURE REVIEW}

In [8], Face acknowledgement has much accepted interest as a broadly received biometric, since collecting samples of a person, with or without subject's intension is easier. This constitutes a wide group of technologies which all work with face but use different scanning techniques. Our visual performance is very robust against a variety of factors such as changes in facial expression, illumination, size or head posture, background, or partial occlusion of a face. The work described by a face acknowledgement system consisting of face image identification, standardization, pre-processing, trait extraction and input to neural network \& matching that execute accurately and effectively on a personal computer platform.

Face acknowledgement is a pattern recognition problem, so comparison between the faces will be done with the help of training/learning algorithm. For 2D recognition, Linear/Nonlinear Projection methods and Artificial Neural Networks are used. The Linear/Nonlinear Projection methods are Principal component analysis(PCA), Linear Discriminant Analysis (LDA), and Gabor .

Linear/Nonlinear Prediction methods generate attribute vector for each human, then categorize the input human inside the database. Producing feature vector also has importance to reduce dimension of the input images. One approach applies image improvement to repress the bad lighting condition before acknowledgement process.

Image enhancements are known as logarithm transform and normalization. Then in [9], feature extraction done with Gabor Wavelets. Finally, using Fisher Face, input face is classified. Song et al. [9] apply a different approach on image preprocessing/enhancement. For pre-processing before attribute 
extraction, calculates the illustration difference between right and left part of face. If there is a large amount of dissimilarities then take the mirror of average illuminated part. When face image is pre-processed, feature extraction is done with PCA. Classification of feature vector is completed with Euclidian Distance. Other accomplishment uses [7] Layered Linear Discriminate Analysis (LDA) to classify faces, and the welfare of Layered LDA is using small sample size database. Widening the attribute extraction can enhance the performance of the system i.e. applying Gabor wavelet, Chief Component Analysis and then, Independent Component Analysis (ICA) on face image. After feature extraction is applied, then cosine sameness measures and the nearby categorisation rule is used to identify. Another method uses facial distances for input vector.

Faces will be forwarded to $\mathrm{NN}$ in the form of a feature extraction or vectored matrix image. The feature extraction image algorithm can have more performance because of using small dimension vector. As network, RBNN [4], and Multilayer Cluster NN are used and as attribute extractor, Discrete Wavelet Transform, DCT, PCA are used. Wavelet $\mathrm{NN}$ uses transfer function which is got from wavelet function series which avoids the concealment in structure design of FFNN [6]. On the other hand, instead of using gray scale images, coloration images can be used as input to the FFNN. R, G, B channels are merged inputs and network supply with a colour info for trouble-free discrimination. Analyzer also works on effects of using different type of networks and feature extractors.

\section{METHODOLOGY}

\subsection{Two Dimensional Discrete Cosine \\ Transform}

In [1], Compression is a process by which the description of computerized documentation is changed so that the capacity required accumulating or the bit-rate required to transmit it is minimised. The DCT is a transform which transmutes a signal or picture from the spatial domain to the basic frequency domain. Lower frequencies are more apparent in an image than higher prevalence where an image is converted into its frequency integrants and higher frequency collaterals are relinquished, the amount of data needed to describe the image without giving up too much image quality will condense.

\subsection{Principal Component Analysis}

In [2], PCA is a useful analytical technique that has established application in fields such as face recognition and minimisation of image size, and is a prevalent technique to find patterns of high dimension data. It is a way of recognising patterns in data, and demonstrates the data in a way to pinnacle their affinities and variances. The other main perk of PCA is that once you have found the patterns in the data, and you compress the size of the data, i.e. by reducing the dimensions of the data, with minimum loss of information.

\subsection{Self Organizing Map}

In [10], the elementary objective of self-organizing maps is to transform an approaching signal pattern of arbitrary aspect into a one or two-dimensional discrete map, and to execute this transformation adaptively in a topologically sequenced fashion. Self-organizing maps attain to recognize batch of identical input vectors in such a way that neurons physically near each other in the neuron layer respond to identical input vectors. They provide a quantization of the image test pieces into a topological space where inputs that are close by in the original space are also nearby in the output space, thereby providing reduction in dimensions and permanence to minor changes in the image sample.

\section{PROCEDURE}

Step 1: Collect all face images dates.

Step 2: Using Adobe Photoshop Pre-process all face images.

Step 3: Import all face images into MATLAB.

Step 4: Using 2D discrete cosine transform all face images.

Compress

Step 5: Design a Self-Organizing Map (SOM) neural network in MATLAB.

Step 6:Input all face images into SOM Neural network.

Step 7: Give training to the neural network and replicating it for different input face images.

Step 8: Apply the steps 1 to step 6 on all face images with the help of principal component analysis instead of $2 \mathrm{D}$ discrete cosine transform.

Step 9: Finally compare the training time of both Principal component Analysis and 2D Discrete Cosine Transform methods and analyze the results.

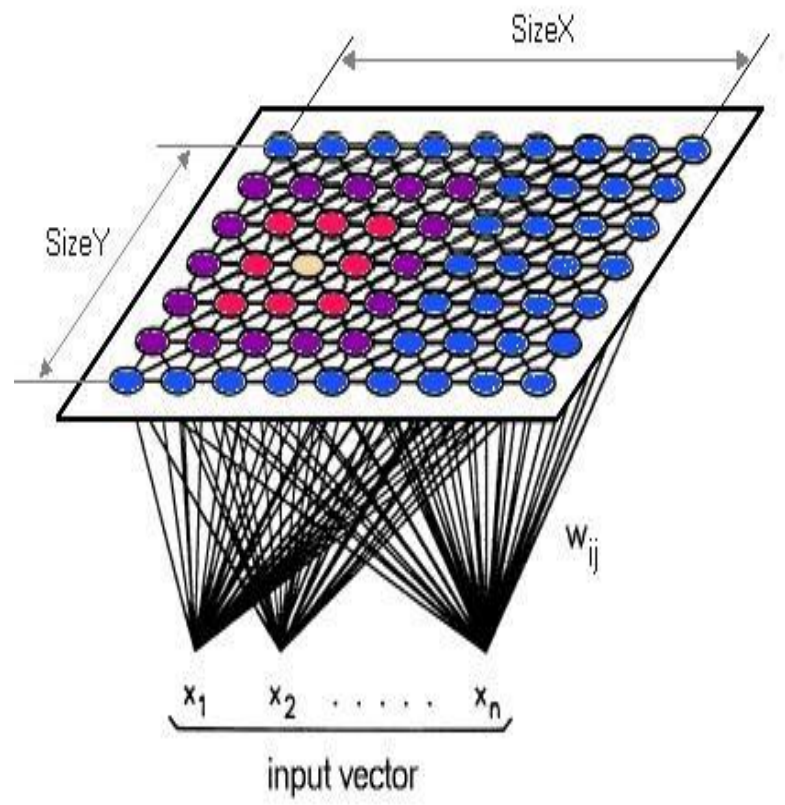

Fig.1 Self Organizing map 


\section{RESULTS AND DISCUSSION}

The face training images are shown in figure 2 .

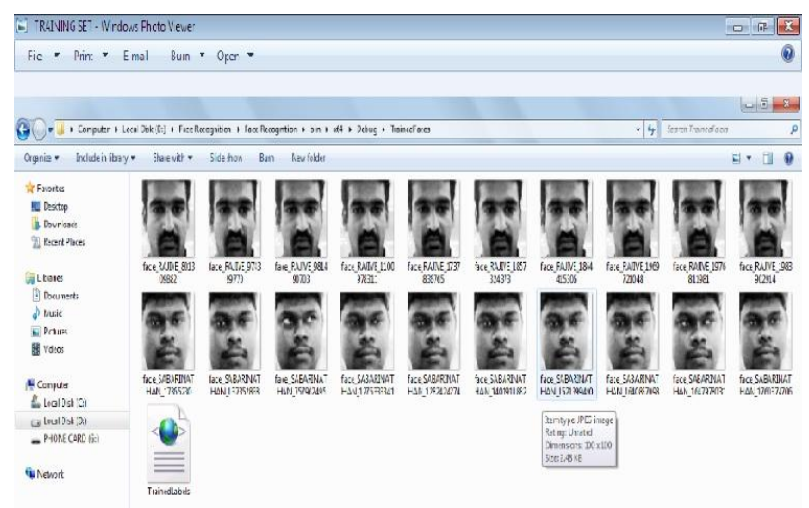

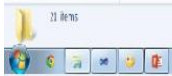

Fig 2 Trained Face images with SOM Network

The untrained input matched with the closest image of the same subject in the training database, generating a correct answer. The output is shown in fig. 3 and 4.

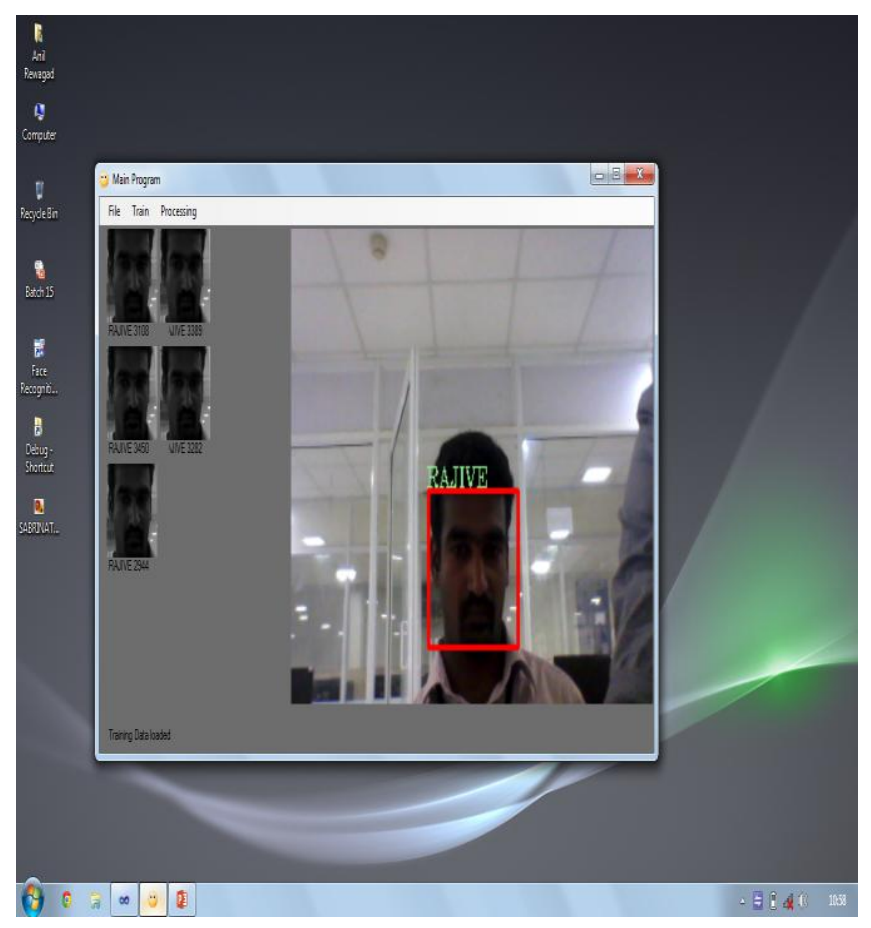

Fig 3. Recognized face from the trained images for First object.

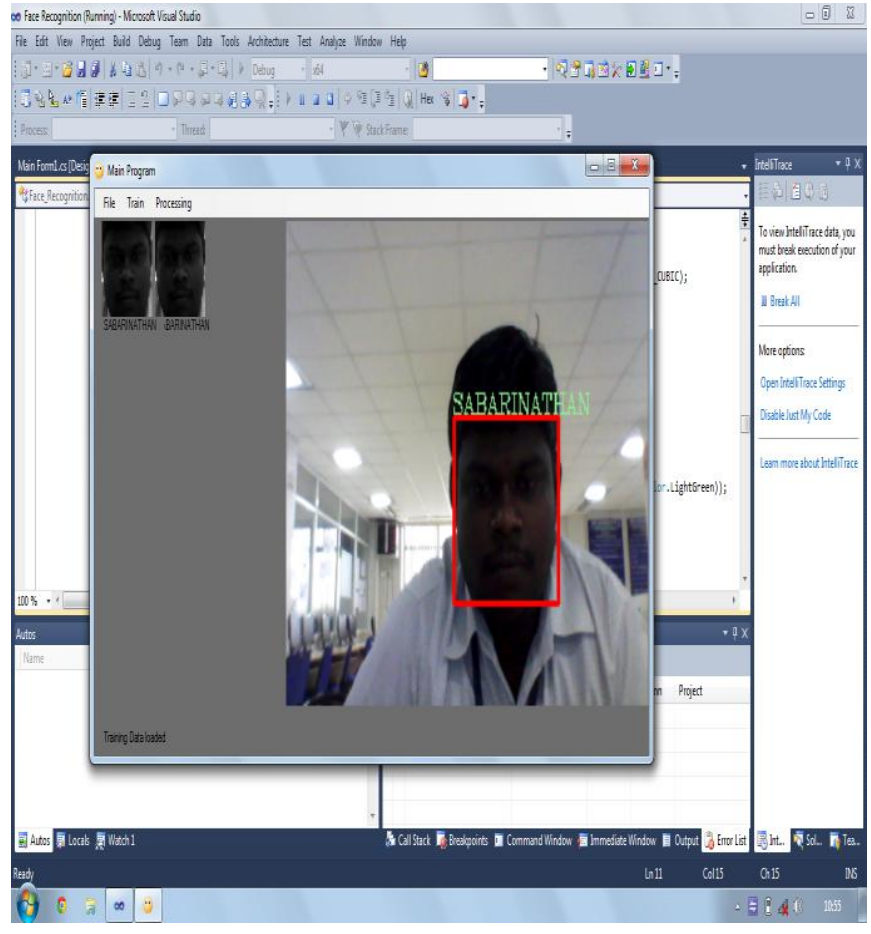

Fig 4. Recognized face from the trained images for Second object.

\begin{tabular}{|c|c|c|l|}
\hline $\begin{array}{l}\text { No. of } \\
\text { epochs }\end{array}$ & $\begin{array}{l}\text { No. of } \\
\text { images }\end{array}$ & $\begin{array}{l}\text { Training time } \\
\text { with 2D- } \\
\text { DCT (sec.) }\end{array}$ & $\begin{array}{l}\text { Training time } \\
\text { with PCA } \\
\text { (sec.) }\end{array}$ \\
\hline 100 & 9 & 1.7277 & 1.1710 \\
\hline 300 & 9 & 3.5705 & 3.1796 \\
\hline 500 & 9 & 5.7956 & 5.0664 \\
\hline 1000 & 9 & 11.3543 & 10.0418 \\
\hline
\end{tabular}

Table 1 Comparison B/w Training Time of Images with 2D-DCT and Principal Component Analysis

\section{CONCLUSION}

The main goal of this paper is achieved by designing of an efficient high-speed face recognition system using neural network. The PCA and the SOM neural network are important for the design and implementation, which are the final algorithms used for the design of an efficient highspeed face recognition system. Principle component analysis with SOM is the efficient technique than 2D-Discrete Cosine Transform with SOM. 


\section{REFERENCES}

[1] Andrew B. Watson. "Image Compression using the discrete Cosine Transform", NASA Ames Research Center, Florida, United States of America. Mathematica Journal, 4(1),1994. p.81-88.

[2] Boualleg A.H, Bencheriet.Ch, Tebbikh H, 2006, “ Automatic Face Recognition using Neural NetworkPCA", Proc. $2^{\text {nd }}$ International and Communication Technologies ICTTA '06, pp. 1920-1925, Damascus, Syria.

[3] Jahan Zeb, Muhammed Yonus Javed and Usman Qayyum, "Low resolution single neural network based face recognition" International journal of Biomedical Sciences. Volume 2, Number 3, 2007, ISSN 1306-1216, pp 206-210.

[4] Kiminori Sato, Shishir Shah, J.K. Aggarwal, "Partial Face Recognition using Radial Basis Function Networks" IEEE journal.

[5] Mandal B, Jiang X D and Kot A, “ Multi-scale Feature Extraction for face recognition” IEEE, 2006.

[6] Pritha D N, Savitha L, Shylaja S S, 2010, "Face Recognition by Feed Forward Neural Network using
Laplacian of Gaussian filter and singular value Decomposition", Proc. 2010. First International conference on Integrated Intelligent computing, pp.5661, Bangalore, India.

[7] Razzak M I, Khan M K, Alghathbar K, and Yousaf R, 2010, “ face Recognition using Layered Linear Discriminant Analysis and small Subspace”, Proc. 2010 $10^{\text {th }}$ IEEE International Conference on Computer and Information Technology (CIT 2010). Pp.1407-1412, West Yorkshire, UK.

[8] Tolba A S, El-Baz A H, El Harby, " Face Recognition: A literature Review", International Journal of Signal Processing, Volume 2, number 1, 2005, ISSN 13044475, pp 88103.

[9] Wang G and Zou, 2006, "Face Recognition based on Image Enhancement and Gabor Features", Proc. $6^{\text {th }}$ World Conference on Intelligent Control and Automation, pp.9761-9764, Dalian, China.

[10] "Self-Organizing Maps. Wikipedia Source", http://en.wikipedia.org/wiki/Self-organizing map, Retrieved 6 January 2007 